\title{
Efficient Image Matching Algorithms Based on Procedures of Searching for 2D Templates ${ }^{1}$
}

\author{
Alexey V. Nefyodov \\ Scientific Council "Cybernetics" of the Russian Academy of Sciences, \\ 40, Vavilov str., Moscow GSP-1 119991, \\ The Russian Federation \\ anef@mail.ru
}

\begin{abstract}
A model of algorithms based on estimates calculation with 2D support sets (2D-AEC) defines recognition algorithms which could be directly applied to images and their fragments. When one uses these algorithms in practice, a problem arises of decreasing their computational complexity. Some constraints on 2D-AEC algorithms allow one to reduce this problem to the problem of efficient search a binary matrix for a certain template. Efficient two-step procedures of searching for a rectangle were suggested earlier. In this paper conditions are determined under which these procedures have the least complexity among all two-step procedures for rectangle search. This result determines image recognition problems for which the performance of 2D-AEC algorithms based on suggested search procedures cannot be improved by applying other two-step search procedures.
\end{abstract}

\section{Introduction}

Today there are a lot of computationally efficient image recognition algorithms designed for working with feature descriptions or image models. At the same time, efficient recognition algorithms that could be directly applied to images and their fragments also are of certain theoretical and practical interest.

A model of algorithms based on estimates calculation (AEC) was defined in [3]. These algorithms were successfully used to solve many problems of pattern recognition where objects were described by feature sets of 1D nature. In [1], a specification of the AEC model for the problems of image recognition was defined - a model of algorithms based on estimate calculation by using two-dimensional information (2DAEC). The principal property of the $2 \mathrm{D}$-AEC class is that a proximity of recognizing images is evaluating by matching their fragments. The range of the problems of 2DAEC includes enumeration of the types of spatial support sets (the types of image fragments) and definition of the corresponding 2D-AEC subclasses, which allow one to produce efficient implementations of the algorithms.

\footnotetext{
1 This work was partly supported by the Russian Foundation for Basic Research, grant nos. 0107-90016, 02-01-00182, and 03-01-06294.
} 
In [2], it is shown that constructing efficient estimate calculation algorithms based on systems of two-dimensional support sets of special form can be reduced to constructing efficient procedures for searching a binary matrix for a certain template (pattern). In the same paper, a formalism describing multistep procedures for searching binary matrices for templates is suggested and two efficient two-step procedures of searching for a rectangle are considered.

In this paper, we present a new result concerning the efficiency of the two-step procedures of searching for a rectangle introduced in [2]. It is proved that, for some problems of searching for a rectangle, suggested procedures have the least computational complexity among all two-step procedures of solving this task. This result shows that, for some image recognition problems, the performance of 2D-AEC algorithms based on suggested search procedures cannot be improved by applying other two-step search procedures. In Section 2, the 2D-AEC model is briefly described. In Section 3, necessary definitions connected with the multistep search procedures are given. In Section 4, effective two-step procedures for rectangle search are introduced and a theorem connected with their optimality is stated.

\section{Model of 2D-AEC Recognition Algorithms}

The 2D-AEC model describes the structure of recognition algorithm and parameters necessary for choosing particular algorithm in the model. 2D-AEC algorithm classifies image $I$ in two stages. On the first stage, a recognizing operator of the algorithm is used to calculate a set of estimates $\left(\Gamma_{1}(I), \Gamma_{2}(I), \ldots, \Gamma_{l}(I)\right)$, where $l$ is a number of classes and $\Gamma_{j}(I)$ is some real number. On the second stage, the information vector $\left(\alpha_{1}, \alpha_{2}, \ldots, \alpha_{l}\right), \alpha_{j} \in\{0,1, \Delta\}$, is constructed from this set with the help of decision rule of the algorithm. Here, $\alpha_{j}=0$ if an algorithm does not assign image $I$ to $j$ th class; $\alpha_{j}=1$, if an algorithm assigns image $I$ to $j$ th class; and $\alpha_{j}=\Delta$, if an algorithm cannot classify image $I$ over $j$ th class.

To define a recognizing operator, it is necessary to assign a system of support sets, proximity function, feature weights, and precedent weights [2]. Let us consider these parameters briefly. Suppose that the description of the image is a rectangular matrix $u \times v$ of the pixels values.

1. A system of support sets $\Omega_{A}$ is a totality of subsets of the Cartesian product $\{1,2, \ldots, u\} \times\{1,2, \ldots, v\}$, i.e., $\Omega_{A}=\left\{\Omega_{1}, \Omega_{2}, \ldots, \Omega_{k}\right\}$, where support set $\Omega_{i}$ is a set of pairs $(r, t), r \in\{1,2, \ldots, u\}, t \in\{1,2, \ldots, v\}$. A recognition algorithm uses a system of support sets to determine the matrices' elements that will be matched during image proximity evaluation.

2. Suppose that $I$ and $I^{\prime}$ are two matrices and $\Omega$ is a support set. A proximity function $B_{\Omega}\left(I, I^{\prime}\right)$ defines whether the matched matrices $I, I^{\prime}$ are "close", and only those elements of matrices are matched, which are located at the positions specified in the support set $\Omega$. The proximity function $B_{\Omega}\left(I, I^{\prime}\right)$ takes two values: 1 if the images are close and 0 otherwise. 
3. Weights of the features (pixels) are defined by a set of real numbers $p_{1}, p_{2}, \ldots, p_{u v}, p_{i}>0, i=1,2, \ldots, u v$; weight of the pixel at position $(i, j)$ is equal to $p_{(i-1) v+j}$. The weight of the support set $\Omega$ is denoted by $p(\Omega)$ and defined as the sum of the weights of the positions specified in $\Omega$.

4. Weights of the precedents $I_{1}, I_{2}, \ldots, I_{m}$ (i.e., the images from the training set) are defined by a set of real numbers $\gamma_{1}, \gamma_{2}, \ldots, \gamma_{m}, \gamma_{q}>0, q=1,2, \ldots, m$; weight of the precedent $I_{q}$ is equal to $\gamma_{q}$.

This point concludes the list of the parameters of the recognizing operator of $2 \mathrm{D}$ AEC. The estimate $\Gamma_{j}(I)$ of the image $I$ over the $j$ th class is defined by the equation

$$
\Gamma_{j}(I)=\frac{1}{N} \frac{1}{\left|W_{j}\right|} \sum_{I^{\prime} \in W_{j}} \gamma\left(I^{\prime}\right) \sum_{\Omega \in \Omega_{A}} p(\Omega) B_{\Omega}\left(I, I^{\prime}\right), \quad j=\overline{1, l},
$$

where $N$ is a normalized coefficient and $W_{j}$ is a set of training images of the $j$ th class.

In most practical applications, the system of support sets contains large quantity of support sets. Therefore, equation (1) often has combinatorial complexity and its direct implementation is very time consuming. In [2], it is shown that under certain constraints on the parameters of the 2D-AEC model the problem of calculating the value of the estimate (1) can be reduced to the problem of the search a binary matrix for a certain template. Then, the problem of effective calculating the value of the estimate (1) can be reduced to the problem of the effective search a binary matrix for a template.

\section{Multistep Procedures of Search for a Template}

Suppose that $u$ and $v$ are positive integers, $M(u, v)$ is the Cartesian product $\{1,2, \ldots, u\} \times\{1,2, \ldots, v\}, E^{u \times v}$ is the set of all binary matrices of size $u \times v$. Consider matrices $C=\left(c_{i j}\right) \in E^{u \times v}$ and $\Phi=\left(\varphi_{p q}\right) \in E^{R_{1} \times R_{2}}$ for which $1 \leq R_{1} \leq u, 1 \leq R_{2} \leq v$ and there exist numbers $p_{1}, p_{2}, q_{1}$, and $q_{2}$ such that $\varphi_{p_{1} 1}=\varphi_{p_{2} R_{2}}=\varphi_{1 q_{1}}=\varphi_{R_{1} q_{2}}=1$. We say that the matrix $\Phi$ is correctly superimposed on the matrix $C$ in a position $(i, j) \in M\left(u-R_{1}+1, v-R_{2}+1\right)$ if, for any numbers $p$ and $q$ such that $\varphi_{p q}=1$, we have $c_{i+p-1, j+q-1}=1$. Figures 1(a) and 1(b) show examples of matrices $C$ and $\Phi$. The cells containing unities in these matrices are filled with black and the cells containing zeros, with white. In this example, the template $\Phi$ correctly overlays $C$ only in the position $(2,2)$.

The problem of searching for a matrix $\Phi_{R_{1} \times R_{2}}$ on the matrix $C_{u \times v}$ consists in finding all positions in the matrix $C$ in which $\Phi$ is correctly superimposed on $C$. A solution to this search problem is the matrix $\widetilde{C}=\left(\tilde{c}_{i j}\right) \in E^{\left(u-R_{1}+1\right) \times\left(v-R_{2}+1\right)}$ with the elements defined by the rule: $\tilde{c}_{i j}=1$ if the template $\Phi$ is correctly superimposed on the matrix $C$ in the position $(i, j)$ and $\tilde{c}_{i j}=0$ otherwise. For the example considered above, the matrix $\tilde{C}$ is shown in Fig. 1(c). 


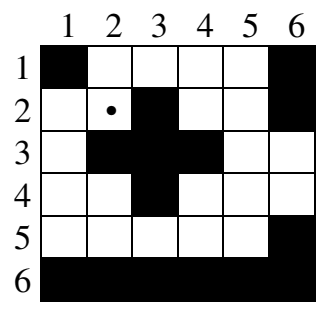

$C_{6 \times 6}$

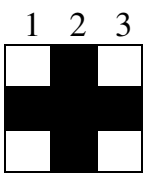

$\Phi_{3 \times 3}$

(b)

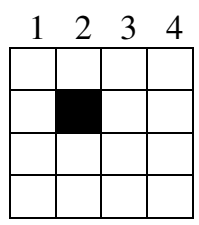

$\tilde{C}$

(c)

Fig. 1. An example of the matrices $C, \Phi$, and $\tilde{C}$.

When dealing with the search problem, we refer to the matrix $\Phi$ as a template. In what follows, we assume that the number of unities in $\Phi$ is no smaller than 2 (i.e., the search problem is nondegenerate).

A multistep search procedure is a method for constructing the matrix $\tilde{C}$ when a matrix $C$ and a template $\Phi$ are given. To construct a multistep search procedure, we must fix the template $\Phi$ and the size of the matrix $C$. A search procedure constructed for given $u, v$, and $\Phi_{R_{1} \times R_{2}}$ can be applied to solve the problem of searching for the template $\Phi$ on an arbitrary matrix $C_{u \times v}$.

The structure of an $n$-step search procedure ( $n$ is a positive integer) is determined by a set of parameters

$$
\left(\left(u_{1}, v_{1}\right),\left(u_{2}, v_{2}\right), \ldots,\left(u_{n-1}, v_{n-1}\right), S_{1}, S_{2}, \ldots, S_{n}\right),
$$

where $u_{k}$ and $v_{k}$ are positive integers,

$$
\begin{gathered}
S_{k}=\left\{S(i, j, k) \mid(i, j) \in M\left(u_{k}, v_{k}\right)\right\}, S(i, j, k) \text { is a subset of } M\left(u_{k-1}, v_{k-1}\right), \\
k=1,2, \ldots, n,
\end{gathered}
$$

and $\left(u_{0}, v_{0}\right)=(u, v),\left(u_{n}, v_{n}\right)=\left(u-R_{1}+1, v-R_{2}+1\right)$. Set of parameters (2) must satisfy four conditions specified later on.

The procedure operates as follows. For a given matrix $C_{0}=\left(c_{i j}^{0}\right) \in E^{u_{0} \times v_{0}}$, the $n$-step search procedure determines a sequence of matrices

$$
C_{1}=\left(c_{i j}^{1}\right)_{u_{1} \times v_{1}}, C_{2}=\left(c_{i j}^{2}\right)_{u_{2} \times v_{2}}, \ldots, C_{n}=\left(c_{i j}^{n}\right)_{u_{n} \times v_{n}},
$$

where $C_{k} \in E^{u_{k} \times v_{k}}$ and $C_{k}=f_{k}\left(C_{k-1}\right)$; here $f_{k}$ is the function defined by the relation 


$$
c_{i j}^{k}=\underset{(p, q) \in S(i, j, k)}{\&} c_{p q}^{k-1},
$$

$i=1,2, \ldots, u_{k} ; j=1,2, \ldots, v_{k} ; k=1,2, \ldots, n$.

Definition 1. Set of parameters (2) determines an $n$-step procedure $F^{n}$ of searching for a template $\Phi_{R_{1} \times R_{2}}$ on a matrix of size $u \times v$ if the following conditions hold:

1) for any $C_{0} \in E^{u_{0} \times v_{0}} C_{n}=\tilde{C}$;

2) $|S(i, j, k)| \geq 2, i=1,2, \ldots, u_{k} ; j=1,2, \ldots, v_{k} ; k=1,2, \ldots, n$;

3) $S\left(i_{1}, j_{1}, k\right) \not \subset S\left(i_{2}, j_{2}, k\right)$ for any admissible $\left(i_{1}, j_{1}\right)$ and $\left(i_{2}, j_{2}\right)$ such that $\left(i_{1}, j_{1}\right) \neq\left(i_{2}, j_{2}\right)$ and for $k=1,2, \ldots, n$;

4) $\bigcup_{(i, j) \in M\left(u_{k}, v_{k}\right)} S(i, j, k)=M\left(u_{k-1}, v_{k-1}\right)$.

Obviously, an $n$-step search procedure $F^{n}$ can be treated as a set of functions

$$
\left(f_{1}, f_{2}, \ldots, f_{n}\right),
$$

where each function $f_{k}$ is determined by the set $S_{k}$ and takes each $C_{k-1} \in E^{u_{k-1} x_{k-1}}$ to $C_{k} \in E^{u_{k} \times v_{k}}$ according to rule (3).

Definition 2. Let $F^{n}$ be an $n$-step search procedure determined by set of parameters (2). A complexity of the procedure $F^{n}$ is the number

$$
\left|F^{n}\right|=\sum_{k=1}^{n} \sum_{(i, j) \in M\left(u_{k}, v_{k}\right)}(|S(i, j, k)|-1) .
$$

The complexity of $F^{n}$ coincides with the number of conjunctions required for implementing this procedure; it is a measure of the computational complexity of the procedure. A procedure for searching for a template $\Phi$ is called optimal in some class $K$ of procedures for searching $\Phi$ if it has the least complexity among all procedures from the class $K$.

\section{Efficient Two-step Procedures for Rectangle Search and their Optimality}

Consider the problem of searching for a rectangle $R_{1} \times R_{2}\left(R_{1} R_{2} \geq 2\right)$ on a $u \times v$ matrix. Let us define a one-step search procedure $F$ for this problem by specifying set of functions (4):

where $f$ is the function defined by the rule

$$
F \leftrightarrow(f),
$$

$$
\begin{gathered}
c_{i j}^{1}=\sum_{(p, q) \in M\left(R_{1}, R_{2}\right)} c_{i+p-1, j+q-1}^{0}, \\
i=1,2, \ldots, u-R_{1}+1, j=1,2, \ldots, v-R_{2}+1 .
\end{gathered}
$$

The complexity of the procedure $F$ is 


$$
|F|=\left(u-R_{1}+1\right)\left(v-R_{2}+1\right)\left(R_{1} R_{2}-1\right) .
$$

Note that there is always a unique one-step procedure of searching for an arbitrary template and this procedure implements an exhaustive search for the template.

The operators defined below are used to construct more computationally effective two-step procedures for rectangle search.

Definition 3. Let $t$ be an integer such that $1 \leq t \leq u$. The mapping $f_{c}(t)$ of the set $E^{u \times v}$ to the set $E^{(u-t+1) \times v}$ that takes each matrix $C=\left(c_{i j}\right) \in E^{u \times v}$ to the matrix $C^{\prime}=\left(c_{i j}^{\prime}\right) \in E^{(u-t+1) \times v}$, where $c_{i j}^{\prime}=c_{i, j} \cdot c_{i+1, j} \cdot \ldots \cdot c_{i+t-1, j}$, is called an operator of column compression in $E^{u \times v}$.

An operator of compression of rows in $E^{u \times v}$ is introduced similarly. Namely, if $t$ is an integer such that $1 \leq t \leq v$, then the mapping $f_{r}(t)$ of the set $E^{u \times v}$ to the set $E^{u \times(v-t+1)}$ that takes each matrix $C=\left(c_{i j}\right) \in E^{u \times v}$ to the matrix $C^{\prime}=\left(c_{i j}^{\prime}\right) \in E^{u \times(v-t+1)}$, where $c_{i j}^{\prime}=c_{i, j} \cdot c_{i, j+1} \cdot \ldots \cdot c_{i, j+t-1}$, is called an operator of row compression in $E^{u \times v}$.

Fig. 2 shows an example of applying, first, a column compression operator to a $6 \times 6$ matrix $C$ and, then, a row compression operator to the resulting matrix. This example shows that the introduced operators may be used for constructing two-step procedures of searching for a rectangle.
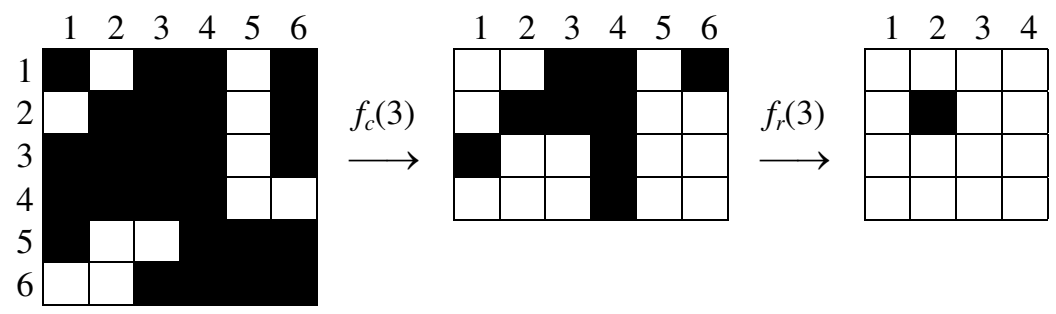

\section{$C_{6 \times 6}$}

Fig. 2. The action of the column and row compression operators.

So, let us define two two-step procedures $F_{c r}$ and $F_{r c}$ for solving the problem of searching for a rectangle $R_{1} \times R_{2}$ on a $u \times v$ matrix by specifying set of functions (4) for each procedure as follows:

It is easy to show that

$$
\begin{aligned}
& F_{c r} \leftrightarrow\left(f_{c}\left(R_{1}\right), f_{r}\left(R_{2}\right)\right), \\
& F_{r c} \leftrightarrow\left(f_{r}\left(R_{2}\right), f_{c}\left(R_{1}\right)\right) .
\end{aligned}
$$

$$
\begin{aligned}
& \left|F_{c r}\right|=\left(u-R_{1}+1\right) v\left(R_{1}-1\right)+\left(u-R_{1}+1\right)\left(v-R_{2}+1\right)\left(R_{2}-1\right), \\
& \left|F_{r c}\right|=u\left(v-R_{2}+1\right)\left(R_{2}-1\right)+\left(u-R_{1}+1\right)\left(v-R_{2}+1\right)\left(R_{1}-1\right) .
\end{aligned}
$$

Note that $\left|F_{c r}\right| \leq\left|F_{r c}\right|$ if $u-R_{1} \leq v-R_{2}$ and

$$
\begin{aligned}
& |F|-\left|F_{c r}\right|=\left(u-R_{1}+1\right)\left(R_{1}-1\right)\left(R_{2}-1\right)\left(v-R_{2}\right), \\
& |F|-\left|F_{r c}\right|=\left(v-R_{2}+1\right)\left(R_{1}-1\right)\left(R_{2}-1\right)\left(u-R_{1}\right) .
\end{aligned}
$$


The last equations indicate that the complexity of the introduced two-step procedures for rectangle search is less than a complexity of the one-step procedure which implements an exhaustive rectangle search.

Consider the problem of searching for a rectangle $R_{1} \times R_{2}$ on a matrix of size $R_{1} \times v$, where $v>R_{2}$. We call such a search problem one-dimensional.

Theorem. Suppose that in one-dimensional problem of searching for a rectangle $R_{2}$ $\leq R_{1}$ and $v \geq 2 R_{2}-1$. Then procedure $F_{c r}$ is optimal in the class of all two-step procedures for solving this problem.

Note that if $R_{2} \leq R_{1}$ and $v<2 R_{2}-1$, then some modification of procedure $F_{c r}$ is optimal in the class of all two-step procedures for solving this problem.

This theorem shows that if one has images represented by $R_{1} \times v$ matrices and recognition algorithm from the 2D-AEC model that matches images by $R_{1} \times R_{2}$ rectangular fragments where $R_{2}<v$ and $R_{2} \leq R_{1}$, then search procedures (5) may be used for effective implementation of this algorithm.

\section{Conclusion}

A model of algorithms based on estimates calculation with 2D support sets (2D-AEC) defines recognition algorithms, which could be directly applied to images and their fragments. The two-step search procedures considered in this paper make it possible to develop efficient estimate calculation algorithms that match images by rectangular fragments. It is shown, for which problems of image recognition the performance of 2D-AEC algorithms based on suggested search procedures cannot be improved by applying other two-step search procedures.

\section{References}

1. Gurevich, I.B.: Determining a Class of Algorithms of Estimates Calculation Based on TwoDimensional Information for Image Recognition. In: Methods and Means of Processing Graphic Information, Intercollegiate Collection of Papers, Yu.G. Vasin, (ed.), Gorky: Gorky State Univ. (1986) 47-66 (in russian)

2. Gurevich, I.B., Nefyodov, A.V.: Algorithms for Estimates Calculations Designed for the Case of 2D Support Sets. Part 1: Rectangular Support Sets. Pattern Recognition and Image Analysis, 4, Vol. 11 (2001) 662-689

3. Zhuravlev, Yu.I., Nikiforov, V.V.: Recognition Algorithms Based on Estimates Calculation. Kibernetika, 3 (1971) 1-11 (in russian) 